\title{
COL6A3 polymorphisms were associated with lung cancer risk in a Chinese population
}

\author{
Ying Duan', Gaowen Liu², Yao Sun ${ }^{3}$, Jiamin Wư ${ }^{3}$, Zichao Xiong ${ }^{3}$, Tianbo $\mathrm{Jin}^{3}$ and Mingwei Chen ${ }^{\text {1* }}$
}

\begin{abstract}
Background: Lung cancer is one of the leading cause of cancer-related death in the world. Recently, many clinical researches have reported that COL6A3 had strong role in many diseases. The aim of this study was to evaluate the association between single nucleotide polymorphisms (SNPS) in COL6A3 and lung cancer susceptibility.

Method: Eight variants in COL6A3 were genotyped in a Chinese Han population including 510 cases and 495 controls using Agena MassARRAY. Genetic models and haplotype analyses were used to calculate the association between COL6A3 SNPs and lung cancer risk. And we assessed the relative risk by the odds ratio (OR) and 95\% confidence interval $(\mathrm{Cl})$.

Results: In our results, we observed that rs115510139 was linked to an increased risk of lung cancer in the codominant (adjusted $\mathrm{OR}=1.61,95 \% \mathrm{Cl}: 1.14-2.27, p=0.007$ ), dominant (adjusted $\mathrm{OR}=1.36,95 \% \mathrm{Cl}: 1.02-1.83, p=$ 0.037 ), recessive (adjusted $\mathrm{OR}=1.41,95 \% \mathrm{Cl}: 1.07-1.85, p=0.015$ ), and log-additive (adjusted $\mathrm{OR}=1.27,95 \% \mathrm{Cl}: 1.07-$ $1.51, p=0.006$ ) models. After gender stratification analysis, we found that rs 115510139 , rs3736341 and rs 12052971 were significant in males but were non-significant in females. Rs 115510139 also can increase the risk of lung cancer in the population of age less than 61 years. When analyzed for the association with lung squamous carcinoma, rs13032404, rs115510139 and rs3736341 were related to the risk of lung cancer.
\end{abstract}

Conclusions: Our findings indicated potential associations between COL6A3 polymorphisms and lung cancer risk, which may contribute to the identification of lung cancer patients in a Chinese population.

Keywords: Lung cancer, Susceptibility, Agena MassARRAY technology, Case-control study, COL6A3, Single nucleotide polymorphisms

\section{Background}

Globally, lung cancer is one of the most common types of incident cancer and the leading cause of cancerrelated death $[1,2]$. It is mainly divided into small cell lung cancer (SCLC) and non-small cell lung cancer (NSCLC), whose mainly clinical manifestation is NSCLC. According to the morphological characteristics of tumor cells under the microscope, NSCLC usually treated with platinum-based chemotherapy [3] was consisted of squamous cell carcinoma, adenocarcinoma, large-cell carcinoma and so on. In addition, lung cancer

\footnotetext{
* Correspondence: chenmw36@163.com

'Department of Respiratory Medicine, The First Affiliated Hospital of School of Medicine of Xi'an Jiao Tong University, \#277 Yanta West Road, Xi'an 710061, Shaanxi, China

Full list of author information is available at the end of the article
}

is the main reason of cancer death for men and the second leading cause of cancer death for women (second only to breast cancer). Every year, there were about 1.8 million new cases diagnosed with lung cancer and about 1.6 million lung cancer deaths estimated in 2012 [4]. Besides, it is well known that cancer is difficult to cure and has a poor prognosis with a 5-year survival rate of lung cancer populations depending on stage and regional differences [2, 5]. In China, lung cancer is also the most common incident cancer and the main cause of cancer death. It was estimated that about 733.3/100,000 cases were diagnosed with lung cancer and 610.5/100,000 died of lung cancer in 2015 [2]. Moreover, new cases and mortality rates of lung cancer have been increasing year

(c) The Author(s). 2019 Open Access This article is distributed under the terms of the Creative Commons Attribution 4.0 International License (http://creativecommons.org/licenses/by/4.0/), which permits unrestricted use, distribution, and reproduction in any medium, provided you give appropriate credit to the original author(s) and the source, provide a link to the Creative Commons license, and indicate if changes were made. The Creative Commons Public Domain Dedication waiver (http://creativecommons.org/publicdomain/zero/1.0/) applies to the data made available in this article, unless otherwise stated. 
by year, so, it is urgent to study the pathogenesis of lung cancer to prevent and treat it better in the future.

But, chemotherapy resistance makes the treatment of lung cancer patients less effective. According to relevant research, the occurrence of lung cancer may have genetic predisposition [6]. Recently, large-scale genome wide association studies were adopted to identify lung cancer susceptibility genes located in chromosomes 5p15.33, 6p21, 6q23-25, 15q24-25.1, and 13q31.3 [7], suggesting that the regions of these chromosomes were associated with the risk of lung cancer, especially in never-smokers.

The COL6A3 (Collagen Type VI Alpha 3 Chain) gene contains 44 exons and is located in chromosome 2q37.3. COL6A3, encoding the a3(VI) chain, contains two $\mathrm{C}$ terminal Von Willebrand factor type A-like domains, subdomains similar to type III fibronectin repeats, and Kunitz protease inhibitors as well as $6-10 \mathrm{~N}$-terminal Von Willebrand factor type A-like domains, thus contributing to most of the amino-terminal globular domain of the collagen VI heterotrimer. This protein is an extracellular matrix protein found in most tissues and its absence or aberrant formation can result in many diseases, such as Congenital Muscular Dystrophies (CMDs) [8].

In our study, we found that the region in chromosome 2 q37.3 was also related to the risk of lung cancer. In addition, our research firstly discussed that COL6A3 gene was associated with lung cancer risk. We aimed to analyze the genetic association of COL6A3 and lung cancer risk among a Chinese population of Shaanxi Han.

\section{Methods and materials}

\section{Study subjects}

We performed the study in accordance with the Declaration of Helsinki. And the protocol was approved by the Ethics Committee of Shaanxi Provincial Cancer Hospital. Using a case-control design, 510 inpatients with lung cancer and 495 controls were enrolled. All patients were recruited between March 2017 and October 2018 from the Shaanxi Provincial Cancer Hospital (Xi'an City, Shaanxi, China) and they were diagnosed with lung cancer, and they had no history of any other cancers. Healthy individuals without any diseases collected from health examination center were used as controls. All patients and controls were a Chinese Han population. And written informed consent was obtained from all individual participants. In total, we collected 1005 peripheral venous blood samples using vacutainer tubes containing EDTA, which were then stored at $-80^{\circ} \mathrm{C}$ refrigerator for DNA extraction experiments.

\section{SNP selection and genotyping}

In total, we successfully chose eight variants (rs1050785, rs7436, rs13032404, rs115510139, rs2645765, rs3736341, rs12052971, rs6720283) in the global population of the
1000 Genome Projects (http://www.internationalgenome.org/). Each SNP had a minor allele frequency (MAF) $>5 \%$. RegulomeDB (http://www.regulomedb.org/) and HaploReg (https://pubs.broadinstitute.org/mammals/haploreg/haploreg.php) were utilized to predict SNP function. According to the manufacturer's instructions of the GoldMag-Mini Whole Blood Genomic DNA Purification Kit (GoldMag. Co. Ltd., Xi'an, China), we extracted genomic DNA from the blood samples. Then, DNA concentration and purity were checked using a spectrophotometer (NanoDrop 2000; Thermo Fisher Scientific, Waltham, MA, USA). Primers for amplification and extension of SNPs were designed by using the Agena Bioscience Assay Design Suite V2.0 software (https://agenacx.com/online-tools/) (Additional file 4: Table S1). Then, we performed SNP genotyping with the Agena MassARRAY platform with iPLEX gold chemistry (Agena Bioscience, San Diego, CA, USA), and managed and analyzed data with Agena Bioscience TYPER, Version 4.0 [9].

\section{Analysis of COL6A3 expression}

We observed the differences in COL6A3 expression between normal lung tissues and lung cancer tissues based on the UALCAN database (http://ualcan.path.uab.edu/ index.html), which is an interactive web resource for analyzing the transcriptome data of cancer. And the Kaplan-Meier Plotter database (http://kmplot.com/analysis/index.php?p=background) included the information downloaded from GEO (Gene Expression Omnibus), EGA (European Genome-phenome Archive) and TCGA (The Cancer Genome Atlas) database was used to analyze the prognostic value of a specific gene. The correlation between the expression of COL6A3 in lung cancer and the overall survival rate was displayed by a Kaplan-Meier survival plot, the hazard ratio (HR) with 95\%CIs and $\log$-rank $p$ value.

\section{Statistical analysis}

The Student's t-test and Pearson's test were applied to evaluate differences in the distribution of age and gender between two groups (cases and controls), respectively. The genotype frequencies among the controls were evaluated departure from Hardy-Weinberg Equilibrium (HWE). And we calculated the association between SNPs and lung cancer risk based on the four model analyses (codominant, dominant, recessive, and logadditive) using logistic regression analysis provided by the PLINK software (version 1.07) [10]. Additionally, Haploview software (version 4.2) was used to generate a linkage disequilibrium (LD) map to observe the degree of linkage between these eight SNPs. All $p$-values were two-tailed and $p$-values less than 0.05 were considered statistically significant. 


\section{Results}

\section{Characteristics of cases and controls}

The case group consisted of 510 inpatients with lung cancer. Three hundred forty-six were males and 149 were females, with mean age $60.78 \pm 9.958$ years. And 495 volunteers (355 males and 155 females) were used as the controls, with mean age $61.94 \pm 7.723$ years. The basic information of eight COL6A3 polymorphisms was displayed in Table 1. The genotype distribution of all eight SNPs in the control group was in accordance with HWE $(p>0.05)$. The frequency distribution of allele " $C$ " of rs115510139 was significantly different between cases and controls $(p=0.006)$, from which we found it to be associated with an increased risk of lung cancer $(\mathrm{OR}=$ 1.28, 95\%CI: 1.08-1.53) in the Chinese Han population. The RegulomeDB Score and HaploReg were used to evaluate the function of the SNPs listed in Additional file 8: Table S5.

\section{Genetic model analysis between COL6A3 variants and lung cancer risk}

To further explore the correlations between COL6A3 variants and lung cancer risk, genetic models (codominant, dominant, recessive, and additive) were applied to this study (Table 2). We observed that the genotypes " $\mathrm{T} /$ $\mathrm{A}-\mathrm{T} / \mathrm{T}$ " of rs115510139 were linked to an increased risk of lung cancer than genotype "A/A" in the dominant model with or without adjustment for gender and age $(\mathrm{OR}=1.36,95 \% \mathrm{CI}: 1.01-1.82, p=0.040$; adjusted $\mathrm{OR}=$ 1.36, 95\%CI: $1.02-1.83, p=0.037)$. Genotype "TT" of rs115510139 had an increased risk of lung cancer in the codominant $(\mathrm{OR}=1.60,95 \% \mathrm{CI}: 1.13-2.25, p=0.008)$ and recessive $(\mathrm{OR}=1.39,95 \% \mathrm{CI}: 1.06-1.83, p=0.018)$ models without adjustment. After adjustment for gender and age, the positive effect of the genotype "TT" in the two models still existed (adjusted $\mathrm{OR}=1.61,95 \% \mathrm{CI}$ : $1.14-2.27, p=0.007$; adjusted $\mathrm{OR}=1.41$, 95\%CI: $1.07-$ $1.85, p=0.015)$. In addition, the log-additive model showed that there was significantly increased association between rs115510139 and lung cancer risk with or without adjustment for gender and age $(\mathrm{OR}=1.27,95 \% \mathrm{CI}$ : $1.07-1.50, p=0.007$; adjusted $\mathrm{OR}=1.27$, 95\%CI: $1.07-$ $1.51, p=0.006)$.

\section{Stratification analysis by gender}

Furthermore, after a stratified analysis by gender, we found that the two variants (rs115510139 and rs3736341) were significant in males but were nonsignificant in females based on the allele model (Table 3) . Chi-square test showed that the frequencies distribution of minor allele of the two variants were significantly different between the controls and the male patients ( $p=0.003$ and 0.038), respectively. Rs115510139-T conferred a significantly higher likelihood of lung cancer risk than the $\mathrm{C}$ allele. In the codominant model, the homozygous genotype "TT" of rs115510139 (adjusted $\mathrm{OR}=1.86 ; 95 \% \mathrm{CI}, 1.23-2.83 ; p=0.003)$ increased the risk of lung cancer by 1.86 -fold. There were significantly increased association between rs115510139 and lung cancer susceptibility in the dominant (adjusted OR= 1.53, 95\%CI: 1.07-2.18, $p=0.018$ ), recessive (adjusted $\mathrm{OR}=1.53,95 \% \mathrm{CI}: 1.10-2.12, p=0.012)$, and log-additive (adjusted $\mathrm{OR}=1.37, \quad 95 \% \mathrm{CI}: \quad 1.11-1.68, \quad p=0.003$ ) models, respectively.

In addition, heterozygous genotype " $\mathrm{T} / \mathrm{C}$ " of rs3736341 (adjusted OR $=0.69$, 95\%CI: 0.49-0.95, $p=$ 0.025 ) showed a protective effect on the risk of lung cancer in the codominant model in males. And there was significant association between rs3736341 and lung cancer risk in the dominant (adjusted $\mathrm{OR}=0.69,95 \% \mathrm{CI}$ : $0.51-0.94, p=0.019$ ) and log-additive (adjusted $\mathrm{OR}=$ 0.69, 95\%CI: 0.49-0.95, $p=0.025)$ models. Genotype "G/ A" of rs12052971 (adjusted OR $=0.72$, 95\%CI: 0.51-0.99, $p=0.048$ ) showed that a significantly decreased association with genetic predisposition of lung cancer in the codominant model. But, non-significant associations

Table 1 Basic information and allele frequencies of the SNPs in COL6A3

\begin{tabular}{|c|c|c|c|c|c|c|c|c|c|}
\hline \multirow[t]{2}{*}{ SNP } & \multirow[t]{2}{*}{ Chr } & \multirow[t]{2}{*}{ Gene } & \multirow{2}{*}{$\begin{array}{l}\text { Alleles } \\
\mathrm{A}<\mathrm{B}\end{array}$} & \multirow[t]{2}{*}{ Role } & \multicolumn{2}{|c|}{$\operatorname{MAF}(A)$} & \multirow{2}{*}{$\begin{array}{l}\text { HWE } \\
p \text {-value }\end{array}$} & \multirow{2}{*}{$\begin{array}{l}\text { OR } \\
(95 \% \mathrm{Cl})\end{array}$} & \multirow[t]{2}{*}{$P^{*}$} \\
\hline & & & & & Case & Control & & & \\
\hline rs1050785 & $2 \mathrm{q} 37.3$ & COL6A3 & $\mathrm{G} / \mathrm{T}$ & 3'-UTR & 0.469 & 0.492 & 0.654 & $0.91(076-1.09)$ & 0.296 \\
\hline rs7436 & $2 \mathrm{q} 37.3$ & COL6A3 & $\mathrm{A} / \mathrm{T}$ & 3'-UTR & 0.171 & 0.182 & 0.764 & $0.92(0.73-1.16)$ & 0.495 \\
\hline rs13032404 & $2 \mathrm{q} 37.3$ & COL6A3 & $A / G$ & Intron & 0.387 & 0.367 & 0.148 & $1.09(0.91-1.30)$ & 0.359 \\
\hline rs115510139 & $2 \mathrm{q} 37.3$ & COL6A3 & $\mathrm{A} / \mathrm{T}$ & Intron & 0.558 & 0.496 & 0.241 & $1.28(1.08-1.53)$ & 0.006 \\
\hline rs2645765 & $2 q 37.3$ & COL6A3 & $A / G$ & Intron & 0.280 & 0.297 & 0.386 & $0.92(0.76-1.12)$ & 0.393 \\
\hline rs3736341 & $2 q 37.3$ & COL6A3 & $\mathrm{C} / \mathrm{T}$ & Intron & 0.247 & 0.273 & 0.131 & $0.87(0.71-1.07)$ & 0.195 \\
\hline rs12052971 & $2 q 37.3$ & COL6A3 & $A / G$ & Intron & 0.250 & 0.249 & 0.103 & $1.00(0.82-1.23)$ & 0.976 \\
\hline rs6720283 & $2 q 37.3$ & COL6A3 & $A / G$ & Intron & 0.459 & 0.447 & 0.466 & $1.05(0.88-1.25)$ & 0.606 \\
\hline
\end{tabular}

95\% Cl 95\% confidence interval, HWE Hardy-Weinberg equilibrium, MAF Minor allele frequency, OR Odds ratio, SNP Single-nucleotide polymorphism $p^{*}$ : Calculated by Pearson $x^{2}$ test 
Table 2 Significant COL6A3 variants associated with lung cancer susceptibility

\begin{tabular}{|c|c|c|c|c|c|c|c|c|c|}
\hline \multirow[t]{2}{*}{ Gene } & \multirow[t]{2}{*}{ SNP } & \multirow[t]{2}{*}{ Model } & \multirow[t]{2}{*}{ Genotype } & \multirow[t]{2}{*}{ Control } & \multirow[t]{2}{*}{ Case } & \multicolumn{2}{|l|}{ Unadjusted } & \multicolumn{2}{|c|}{ Adjusted for Gender and Age } \\
\hline & & & & & & OR $(95 \% \mathrm{Cl})$ & $p^{\mathrm{a}}$-value & OR $(95 \% \mathrm{Cl})$ & $p^{\mathrm{b}}$-value \\
\hline \multirow[t]{8}{*}{ COL6A3 } & rs115510139 & Codominant & $\mathrm{A} / \mathrm{A}$ & $131(26.7 \%)$ & $108(21.2 \%)$ & 1.00 & & 1.00 & \\
\hline & & & $A / T$ & $232(47.3 \%)$ & $235(46.1 \%)$ & $1.23(0.90-1.68)$ & 0.197 & $1.23(0.90-1.68)$ & 0.196 \\
\hline & & & $\mathrm{T} / \mathrm{T}$ & $127(26.0 \%)$ & $167(32.7 \%)$ & $1.60(1.13-2.25)$ & 0.008 & $1.61(1.14-2.27)$ & 0.007 \\
\hline & & Dominant & A/A & $131(26.7 \%)$ & $108(21.2 \%)$ & 1.00 & 0.040 & 1.00 & 0.037 \\
\hline & & & T/A-T/T & $359(73.3 \%)$ & $402(78.8 \%)$ & $1.36(1.01-1.82)$ & & $1.36(1.02-1.83)$ & \\
\hline & & Recessive & $\mathrm{A} / \mathrm{A}-\mathrm{A} / \mathrm{T}$ & $363(74.0 \%)$ & $343(67.3 \%)$ & 1.00 & 0.018 & 1.00 & 0.015 \\
\hline & & & $\mathrm{T} / \mathrm{T}$ & $127(26.0 \%)$ & $167(32.7 \%)$ & $1.39(1.06-1.83)$ & & $1.41(1.07-1.85)$ & \\
\hline & & Log-additive & - & - & - & $1.27(1.07-1.50)$ & 0.007 & $1.27(1.07-1.51)$ & 0.006 \\
\hline
\end{tabular}

Bold type indicates statistical significance $(p<0.05)$

$\mathrm{Cl}$ Confidence interval, OR Odds ratio, SNP Single-nucleotide polymorphism

$p^{\text {a }}$ : Calculated by logistic regression analysis

$p^{b}$ : Calculated by logistic regression analysis adjusted for gender and age

Table 3 Significant variants in COL6A3 associated with lung risk in males and females

\begin{tabular}{|c|c|c|c|c|c|c|c|c|c|}
\hline & \multirow[t]{2}{*}{ SNP } & \multirow[t]{2}{*}{ Model } & \multirow[t]{2}{*}{ Genotype } & \multirow{2}{*}{$\begin{array}{l}\text { Control } \\
(\%)\end{array}$} & \multirow[t]{2}{*}{ Case (\%) } & \multicolumn{2}{|c|}{ Adjustment With Age in males } & \multicolumn{2}{|c|}{ Adjustment With Age in females } \\
\hline & & & & & & OR $(95 \% \mathrm{Cl})$ & $p^{\#}$-value & OR $(95 \% \mathrm{Cl})$ & $p^{\#}$-value \\
\hline \multirow[t]{27}{*}{ COL6A3 } & rs115510139 & Allele & $\mathrm{A} / \mathrm{T}$ & - & - & $1.38(1.11-1.70)$ & $0.003^{*}$ & $0.92(0.66-1.26)$ & 0.592 \\
\hline & & Codominant & $A / A$ & $95(27.5)$ & $71(20.0)$ & 1.00 & & 1.00 & \\
\hline & & & $\mathrm{A} / \mathrm{T}$ & $163(47.3)$ & $165(46.5)$ & $1.35(0.93-1.97)$ & 0.118 & $0.83(0.49-1.43)$ & 0.506 \\
\hline & & & $\mathrm{T} / \mathrm{T}$ & $87(25.2)$ & 119 (33.5) & $1.86(1.23-2.83)$ & 0.003 & $0.86(0.46-1.59)$ & 0.624 \\
\hline & & Dominant & $A / A$ & $95(27.5)$ & $71(20.0)$ & 1.00 & 0.018 & 1.00 & 0.497 \\
\hline & & & $\mathrm{A} / \mathrm{T}-\mathrm{T} / \mathrm{T}$ & $250(35.1)$ & $284(80.0)$ & $1.53(1.07-2.18)$ & & $0.84(0.51-1.39)$ & \\
\hline & & Recessive & $\mathrm{A} / \mathrm{A}-\mathrm{A} / \mathrm{T}$ & $258(95.3)$ & $236(66.5)$ & 1.00 & 0.012 & 1.00 & 0.870 \\
\hline & & & $\mathrm{T} / \mathrm{T}$ & $87(25.2)$ & 119 (33.5) & $1.53(1.10-2.12)$ & & $0.96(0.56-1.62)$ & \\
\hline & & Log-additive & - & - & - & $1.37(1.11-1.68)$ & 0.003 & $0.92(0.68-1.26)$ & 0.604 \\
\hline & rs3736341 & Allele & $\mathrm{T} / \mathrm{C}$ & - & - & $0.77(0.60-0.99)$ & $0.038^{*}$ & $1.15(0.80-1.66)$ & 0.437 \\
\hline & & Codominant & $\mathrm{T} / \mathrm{T}$ & $177(53.5)$ & $214(61.7)$ & 1.00 & & 1.00 & \\
\hline & & & $\mathrm{T} / \mathrm{C}$ & $126(38.1)$ & 109 (31.4) & $0.69(0.49-0.95)$ & 0.025 & $1.30(0.79-2.13)$ & 0.306 \\
\hline & & & $\mathrm{C} / \mathrm{C}$ & $28(8.4)$ & $24(6.9)$ & $0.71(0.40-1.27)$ & 0.250 & $1.13(0.51-2.50)$ & 0.766 \\
\hline & & Dominant & $\mathrm{T} / \mathrm{T}$ & $177(53.5)$ & $214(61.7)$ & 1.00 & 0.019 & 1.00 & 0.331 \\
\hline & & & $\mathrm{T} / \mathrm{C}-\mathrm{C} / \mathrm{C}$ & $154(46.5)$ & $133(38.3)$ & $0.69(0.51-0.94)$ & & $1.26(0.79-2.00)$ & \\
\hline & & Recessive & $\mathrm{T} / \mathrm{T}-\mathrm{T} / \mathrm{C}$ & 303 (91.6) & $323(93.1)$ & 1.00 & 0.486 & 1.00 & 0.970 \\
\hline & & & $\mathrm{C} / \mathrm{C}$ & $28(8.4)$ & $24(6.9)$ & $0.82(0.46-1.44)$ & & $1.02(0.47-2.19)$ & \\
\hline & & Log-additive & - & - & - & $0.77(0.61-0.98)$ & 0.034 & $1.14(0.81-1.61)$ & 0.457 \\
\hline & rs12052971 & Allele & $\mathrm{G} / \mathrm{A}$ & - & - & $0.93(0.73-1.18)$ & $0.555^{*}$ & $1.24(0.83-1.86)$ & 0.293 \\
\hline & & Codominant & $\mathrm{G} / \mathrm{G}$ & $193(56.6)$ & $218(61.6)$ & 1.00 & & 1.00 & \\
\hline & & & $\mathrm{G} / \mathrm{A}$ & 119 (34.9) & $98(27.7)$ & $0.72(0.51-0.99)$ & 0.048 & $1.13(0.67-1.89)$ & 0.657 \\
\hline & & & A/A & $29(8.5)$ & $38(10.7)$ & $1.19(0.71-2.01)$ & 0.513 & $1.74(0.63-4.82)$ & 0.284 \\
\hline & & Dominant & $\mathrm{G} / \mathrm{G}$ & $193(56.6)$ & $218(61.6)$ & 1.00 & 0.168 & 1.00 & 0.450 \\
\hline & & & $\mathrm{G} / \mathrm{A}-\mathrm{A} / \mathrm{A}$ & $148(43.4)$ & $136(38.4)$ & $0.81(0.60-1.09)$ & & $1.21(0.74-1.98)$ & \\
\hline & & Recessive & G/G-G/A & $312(91.5)$ & $316(89.3)$ & 1.00 & 0.267 & 1.00 & 0.314 \\
\hline & & & A/A & $29(8.5)$ & $38(10.7)$ & $1.34(0.80-2.23)$ & & $1.67(0.61-4.54)$ & \\
\hline & & Log-additive & - & - & - & $0.94(0.75-1.18)$ & 0.598 & $1.22(0.83-1.81)$ & 0.313 \\
\hline
\end{tabular}

Bold type indicates statistical significance $(p<0.05)$

SNP Single nucleotide polymorphism, OR Odds ratio, $95 \% \mathrm{Cl} 95 \%$ confidence interval

$p^{*}$ : Calculated by Pearson $x^{2}$ test

$p^{\#}$ : $p$-values were calculated by logistic regression analysis with adjustment for age 
were found between rs12052971 and lung cancer risk in any other models.

\section{Stratification analysis by the age of 61 years}

We also observed the correlation between lung cancer susceptibility and COL6A3 variants with stratification analysis by the age of 61 years (Table 4 ). There was not significantly decreased association between rs115510139 and lung cancer risk in the populations of more than 61 years, but it was statistically significant in less than 61year-old populations. The variant rs115510139 was observed to increase lung cancer susceptibility in the codominant $(\mathrm{OR}=1.84,95 \% \mathrm{CI}: \quad 1.12-3.03, \quad p=0.016)$, dominant $(\mathrm{OR}=1.56,95 \% \mathrm{CI}: 1.03-2.38, p=0.037)$, and log-additive $(\mathrm{OR}=1.36,95 \% \mathrm{CI} ; 1.06-1.74, \quad p=0.016)$ models.

\section{Pathological information analysis with lung} adenocarcinoma and lung squamous carcinoma

After analysis for association with lung adenocarcinoma without adjustment (Table 5), we discovered that rs13032404 was significant in the recessive model $(p=$ 0.038). In the other models, the genotype of rs13032404 had no significant relationship with lung cancer risk. When analyzed by association with lung squamous carcinoma, the increase risk of rs13032404 was apparent in the dominant model (adjusted OR $=1.58$, 95\%CI: $1.02-$ 2.47, $p=0.042$ ) after adjustment for gender and age. Besides, rs115510139 and rs3736341 were related to the risk of lung cancer in the four models. As for rs115510139, the significantly increased risk was observed in the codominant (adjusted OR $=1.58,95 \% \mathrm{CI}$ : 1.02-2.47, $p=0.042$ ), dominant (adjusted $\mathrm{OR}=1.89$, 95\%CI: $1.11-3.20, p=0.019$ ), recessive (adjusted $\mathrm{OR}=$ 1.59, 95\%CI: $1.02-2.46, p=0.040$ ), and log-additive (adjusted $\mathrm{OR}=1.48,95 \% \mathrm{CI}: 1.11-1.98, p=0.007)$ models. Inversely, rs3736341 showed the protective effect on the lung cancer susceptibility in the codominant (adjusted
$\mathrm{OR}=0.33,95 \% \mathrm{CI}: 0.11-0.95, p=0.040)$, dominant (adjusted $\mathrm{OR}=0.61,95 \% \mathrm{CI}: 0.40-0.95, p=0.027)$, and $\log$ additive (adjusted OR $=0.63,95 \% \mathrm{CI}$ : 0.44-0.91, $p=$ $0.012)$ models. In addition, we also evaluated the association between COL6A3 SNPs and lung cancer patients with or without lymph node metastasis (Additional file 5: Table S2) as well as the clinical staging of lung cancer patients (Additional file 6: Table S3). But, the three variants did not show a significant correlation with the susceptibility of lung cancer.

\section{LD and haplotype analysis}

We further performed LD analysis among the eight SNPs (rs1050785, rs7436, rs13032404, rs115510139, rs2645765, rs3736341, rs12052971, rs6720283) in COL6A3. A strong linkage mapped to a 10-kb LD block between rs12052971 and rs6720283 was found (Fig. 1). Unfortunately, there were no statistically significant differences between patients and controls among the COL6A3 haplotypes (Additional file 7: Table S4).

\section{Discussion}

In the present case-control study, we investigated the connections of eight variants of COL6A3 and lung cancer risk in a Han population of China. Our results firstly revealed that rs115510139 was significantly associated with lung cancer risk. And when stratified analysis by clinical information, rs13032404, rs115510139, rs3736341, rs12052971 were found to be related with lung cancer.

The COL6A3 gene encodes the alpha- 3 chain of type VI collagen, one of the three alpha chains of type VI collagen. Type VI collagen is structured as a trimer composed of three different alpha chains: alpha-1(VI), alpha$2(\mathrm{VI})$, and alpha-3(VI). The protein is a ubiquitous extracellular matrix protein and usually found in most connective tissues, including muscle, skin, tendon, and vessels. It is now clear that type VI collagen has vital role

Table 4 Significant variants in COL6A3 associated with lung susceptibility after stratified by age of 61 years

\begin{tabular}{|c|c|c|c|c|c|c|c|c|c|}
\hline \multirow[t]{2}{*}{ Gene } & \multirow[t]{2}{*}{ SNP } & \multirow[t]{2}{*}{ Model } & \multirow[t]{2}{*}{ Genotype } & \multirow[t]{2}{*}{ Control } & \multirow[t]{2}{*}{ Case } & \multicolumn{2}{|l|}{ Age $>61$ years } & \multicolumn{2}{|l|}{ Age $\leq 61$ years } \\
\hline & & & & & & OR $(95 \% \mathrm{Cl})$ & $p$-value & OR $(95 \% \mathrm{Cl})$ & $p$-value \\
\hline \multirow[t]{8}{*}{ COL6A3 } & rs115510139 & Codominant & $\mathrm{A} / \mathrm{A}$ & 131 (26.7\%) & $108(21.2 \%)$ & 1.00 & & 1.00 & \\
\hline & & & $\mathrm{A} / \mathrm{T}$ & $232(47.3 \%)$ & 235 (46.1\%) & $0.77(0.51-1.16)$ & 0.212 & $1.40(0.89-2.21)$ & 0.143 \\
\hline & & & $\mathrm{T} / \mathrm{T}$ & $127(26.0 \%)$ & 167 (32.7\%) & $0.71(0.43-1.16)$ & 0.167 & $1.84(1.12-3.03)$ & 0.016 \\
\hline & & Dominant & $\mathrm{A} / \mathrm{A}$ & $131(26.7 \%)$ & $108(21.2 \%)$ & 1.00 & 0.142 & 1.00 & 0.037 \\
\hline & & & T/A-T/T & 359 (73.3\%) & 402 (78.8\%) & $0.75(0.51-1.10)$ & & $1.56(1.03-2.38)$ & \\
\hline & & Recessive & $\mathrm{A} / \mathrm{A}-\mathrm{A} / \mathrm{T}$ & $363(74.0 \%)$ & $343(67.3 \%)$ & 1.00 & 0.393 & 1.00 & 0.055 \\
\hline & & & $\mathrm{T} / \mathrm{T}$ & $127(26.0 \%)$ & 167 (32.7\%) & $0.83(0.55-1.27)$ & & $1.48(0.99-2.22)$ & \\
\hline & & Log-additive & - & - & - & $0.84(0.66-1.07)$ & 0.154 & $1.36(1.06-1.74)$ & 0.016 \\
\hline
\end{tabular}

Bold type indicates statistical significance $(p<0.05)$

SNP Single nucleotide polymorphism, OR Odds ratio, 95\% Cl 95\% confidence interval

$p$ : $p$-values were calculated by logistic regression analysis with adjustment for gender 
Table 5 Significant variants in COL6A3 associated with lung risk in patients with lung adenocarcinoma and lung squamous carcinoma

\begin{tabular}{|c|c|c|c|c|c|c|c|c|c|}
\hline & \multirow[t]{2}{*}{ SNP } & \multirow[t]{2}{*}{ Model } & \multirow[t]{2}{*}{ Genotype } & \multirow[t]{2}{*}{ Control (\%) } & \multirow[t]{2}{*}{ Case (\%) } & \multicolumn{2}{|c|}{ Without Adjustment } & \multicolumn{2}{|c|}{ Adjustment by gender and age } \\
\hline & & & & & & OR $(95 \% \mathrm{Cl})$ & $\overline{p \text {-value }}$ & OR $(95 \% \mathrm{Cl})$ & $p$-value \\
\hline \multicolumn{10}{|c|}{ Lung adenocarcinoma } \\
\hline \multirow[t]{8}{*}{ COL6A3 } & rs13032404 & Codominant & $A / A$ & 95 (27.5) & $71(20.0)$ & 1.00 & & 1.00 & \\
\hline & & & $A / T$ & $163(47.3)$ & $165(46.5)$ & $0.93(0.64-1.35)$ & 0.703 & $0.92(0.63-1.34)$ & 0.672 \\
\hline & & & $T / T$ & $87(25.2)$ & $119(33.5)$ & $1.56(0.95-2.59)$ & 0.768 & $1.46(0.88-2.44)$ & 0.146 \\
\hline & & Dominant & $\mathrm{A} / \mathrm{A}$ & $95(27.5)$ & $71(20.0)$ & 1.00 & 0.018 & 1.00 & 0.869 \\
\hline & & & $\mathrm{A} / \mathrm{T}-\mathrm{T} / \mathrm{T}$ & $250(35.1)$ & $284(80.0)$ & $1.05(0.74-1.49)$ & & $1.03(0.72-1.46)$ & \\
\hline & & Recessive & $\mathrm{A} / \mathrm{A}-\mathrm{A} / \mathrm{T}$ & $258(95.3)$ & $236(66.5)$ & 1.00 & 0.038 & 1.00 & 0.076 \\
\hline & & & $T / T$ & $87(25.2)$ & $119(33.5)$ & $1.63(0.92-2.58)$ & & $1.53(0.96-2.44)$ & \\
\hline & & Log-additive & - & - & - & $1.17(0.92-1.50)$ & 0.204 & $1.14(0.89-1.46)$ & 0.305 \\
\hline \multicolumn{10}{|c|}{ Lung Squamous Carcinoma } \\
\hline \multirow[t]{24}{*}{ COL6A3 } & rs13032404 & Codominant & $\mathrm{A} / \mathrm{A}$ & $95(27.5)$ & $71(20.0)$ & 1.00 & & 1.00 & \\
\hline & & & $\mathrm{A} / \mathrm{T}$ & $163(47.3)$ & $165(46.5)$ & $1.51(0.96-2.36)$ & 0.074 & $1.57(0.99-2.49)$ & 0.054 \\
\hline & & & $\mathrm{T} / \mathrm{T}$ & 87 (25.2) & 119 (33.5) & $1.56(0.82-2.99)$ & 0.177 & $1.64(0.84-3.18)$ & 0.147 \\
\hline & & Dominant & $\mathrm{A} / \mathrm{A}$ & $95(27.5)$ & $71(20.0)$ & 1.00 & 0.059 & 1.00 & 0.042 \\
\hline & & & $\mathrm{A} / \mathrm{T}-\mathrm{T} / \mathrm{T}$ & $250(35.1)$ & $284(80.0)$ & $1.52(0.98-2.34)$ & & $1.58(1.02-2.47)$ & \\
\hline & & Recessive & $\mathrm{A} / \mathrm{A}-\mathrm{A} / \mathrm{T}$ & $258(95.3)$ & $236(66.5)$ & 1.00 & 0.508 & 1.00 & 0.475 \\
\hline & & & $T / T$ & $87(25.2)$ & 119 (33.5) & $1.22(0.68-2.18)$ & & $1.24(0.68-2.26)$ & \\
\hline & & Log-additive & - & - & - & $1.30(0.96-1.76)$ & 0.086 & $1.34(0.98-1.82)$ & 0.064 \\
\hline & rs115510139 & Codominant & $\mathrm{A} / \mathrm{A}$ & 131 (26.7\%) & $108(21.2 \%)$ & 1.00 & & 1.00 & \\
\hline & & & $\mathrm{A} / \mathrm{T}$ & $232(47.3 \%)$ & 235 (46.1\%) & $1.64(0.94-2.84)$ & 0.080 & $1.68(0.96-2.95)$ & 0.007 \\
\hline & & & $\mathrm{T} / \mathrm{T}$ & 127 (26.0\%) & 167 (32.7\%) & $2.17(1.21-3.89)$ & 0.010 & $2.27(1.25-4.13)$ & 0.007 \\
\hline & & Dominant & $\mathrm{A} / \mathrm{A}$ & 131 (26.7\%) & $108(21.2 \%)$ & 1.00 & & 1.00 & \\
\hline & & & T/A-T/T & 359 (73.3\%) & 402 (78.8\%) & $1.83(1.09-3.07)$ & 0.024 & $1.89(1.11-3.20)$ & 0.019 \\
\hline & & Recessive & $\mathrm{A} / \mathrm{A}-\mathrm{A} / \mathrm{T}$ & 363 (74.0\%) & 343 (67.3\%) & 1.00 & 0.047 & 1.00 & 0.040 \\
\hline & & & $\mathrm{T} / \mathrm{T}$ & 127 (26.0\%) & 167 (32.7\%) & $1.54(1.01-2.36)$ & & $1.59(1.02-2.46)$ & \\
\hline & & Log-additive & - & - & - & $1.45(1.10-1.92)$ & 0.010 & $1.48(1.11-1.98)$ & 0.007 \\
\hline & rs3736341 & Codominant & $\mathrm{T} / \mathrm{T}$ & $177(53.5)$ & $214(61.7)$ & 1.00 & & 1.00 & \\
\hline & & & T/C & $126(38.1)$ & $109(31.4)$ & $0.69(0.44-1.07)$ & 0.096 & $0.68(0.43-1.07)$ & 0.098 \\
\hline & & & $\mathrm{C} / \mathrm{C}$ & $28(8.4)$ & $24(6.9)$ & $0.32(0.11-0.91)$ & 0.033 & $0.33(0.11-0.95)$ & 0.040 \\
\hline & & Dominant & $T / T$ & $177(53.5)$ & $214(61.7)$ & 1.00 & 0.025 & 1.00 & 0.027 \\
\hline & & & $\mathrm{T} / \mathrm{C}-\mathrm{C} / \mathrm{C}$ & $154(46.5)$ & $133(38.3)$ & $0.62(0.40-0.94)$ & & $0.61(0.40-0.95)$ & \\
\hline & & Recessive & T/T-T/C & 303 (91.6) & $323(93.1)$ & 1.00 & 0.057 & 1.00 & 0.068 \\
\hline & & & $\mathrm{C} / \mathrm{C}$ & $28(8.4)$ & $24(6.9)$ & $0.36(0.13-1.03)$ & & $0.37(0.13-1.07)$ & \\
\hline & & Log-additive & - & - & - & $0.63(0.44-0.90)$ & 0.010 & $0.63(0.44-0.91)$ & 0.012 \\
\hline
\end{tabular}

$p$-values were calculated by logistic regression analysis with adjustment for gender and age

Bold type indicates statistical significance $(p<0.05)$

SNP Single nucleotide polymorphism, OR Odds ratio, $95 \% \mathrm{Cl} 95 \%$ confidence interval

and can suppress apoptosis and oxidative damage, affect metabolic level, and enhance cell growth. Recent researches focused on genetic studies of lung cancer and revealed some achievements $[11,12]$. Because gene mutations in COL6A1, COL6A2 and COL6A3 have been shown to result in muscular dystrophy, which indicated that collagen VI is particularly necessary for the vitality of skeletal muscle. Further, the article by Lampe AK et al. [8] about collagen VI related muscle disorders describes that mutations in the collagen genes (COL6A1, COL6A2, and COL6A3) can give rise to Bethlem myopathy (BM) and Ullrich congenital muscular dystrophy (UCMD). The recessive COL6A3 mutations (p.R3043H and p.P3082R) can cause neurological disorder early- 


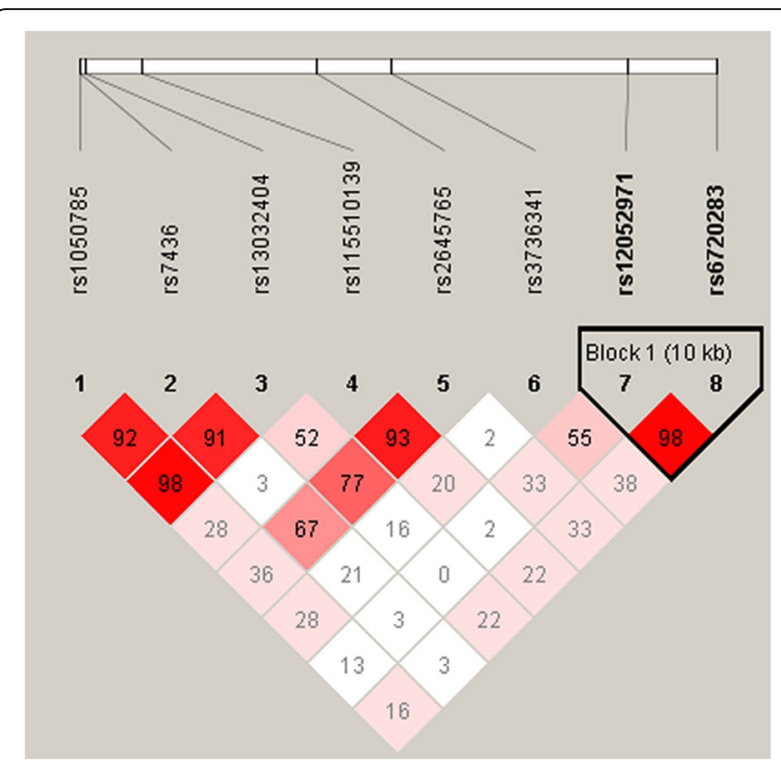

Fig. 1 Linkage disequilibrium (LD) analysis of eight SNPs in COL6A3. The LD value is determined by $r^{2}>0.8$ analyzed by Haploview software 4.2. The number in the diamonds is the LOD score of $r^{2}$. Standard color schemes indicates different levels of LD. Bright red: $L O D>2$, D' = 1; Pink red: $L O D<2$, D' $<$; White: $L O D<2$, $D^{\prime}<1$

onset isolated dystonia $[11,12]$. COL6A3 SNPs were also found to confer susceptibility to early-onset dyslipidemia [13].

Not only that, inhibiting COL6A3 expression was related to insulin resistance and adipose tissue inflammation via suppression of the induction of monocyte chemoattractant protein (MCP1) [14]. And COL6A3 was highly expressed in pancreatic ductal adenocarcinoma (PDA) tissue. In serum levels, its expression was higher associated with perineural invasion and cigarette smoking. And significantly upregulated expression of multiple genes (including COL6A2, and COL6A3) were found in adamantinomatous craniopharyngioma tumor samples [15]. Ran Ao et al. reported that silencing of COL6A3 can inhibit gastric cancer cell proliferation, migration, invasion, and apoptosis by the PI3k-Akt signaling pathway [16]. In addition, COL6A3 played the clinical relevance in the development of colorectal cancer validated by silico analysis of cell type-specific gene expression and COL6A3 knockout experiments [17]. In the database of UALCAN (http://ualcan.path.uab.edu/ index.html) (Additional file 1: Figure S1 and Additional file 2: Figure S2), COL6A3 expression indicated a significant difference between normal lung tissues and lung adenocarcinoma- or lung squamous cell carcinoma tumor-samples. Further, the correlations of survival rates with the expression of COL6A3 in lung cancer illustrated that lung cancer patients with higher COL6A3 expression had a lower survival rates $(\mathrm{HR}=1.32,95 \% \mathrm{CI}$ : 1.11-1.58, log-rank $p=0.0018$, Additional file 3: Figure
S3) shown in the database of Kaplan-Meier Plotter (http://kmplot.com/analysis/index.php?p=service\&cancer=gastric). Hence, COL6A3 was indeed involved in the process of many cancers.

In the article, our results demonstrated that COL6A3 gene was involved in the progress of lung cancer. However, the overall information about the association between COL6A3 polymorphisms and lung cancer risk was few. Thus, a larger sample size and more in-depth analyses will be needed to verify the above results.

\section{Conclusion}

In our results, we observed that rs115510139 was linked to an increased risk of lung cancer in the models analysis with or without adjustment for gender and age. After stratification analysis by gender and age of 61 years, rs115510139 was still associated with lung cancer risk. When analyzed for the association with lung squamous carcinoma, rs13032404, rs115510139 and rs3736341 were related to the risk of lung cancer. Our findings showed potential associations between COL6A3 polymorphisms and lung cancer risk, which may contribute to the identification of lung cancer patients in a Chinese populations.

\section{Additional files}

Additional file 1: Figure S1. Expression of COL6A3 in normal lung tissues and lung adenocarcinoma (LUAD) tissues. There was significant difference between normal lung tissues $(n=59)$ and LUAD tissues $(n=515)(p<0.05)$. Lung adenocarcinoma, LUAD. (TIF $1549 \mathrm{~kb})$

Additional file 2: Figure S2. Expression of COL6A3 in normal lung tissues and lung squamous cell carcinoma (LUSC) tissues. There was significant difference between normal lung tissues $(n=52)$ and LUSC tissues $(n=503)(p<0.05)$. Lung squamous cell carcinoma, LUSC. (TIF $1551 \mathrm{~kb}$ )

Additional file 3: Figure S3. The association between COL6A3 expression and survival rate in lung cancer patients. Lung cancer patients with higher COL6A3 expression had a lower survival rates shown in the database of Kaplan-Meier Plotter. (TIF 910 kb)

Additional file 4: Table S1. PCR primers for amplification and extension of loci used in this study. (DOCX $17 \mathrm{~kb}$ )

Additional file 5: Table S2. Significant variants in COL6A3 associated with lung cancer risk in patients with lymph node metastasis. (DOCX $26 \mathrm{~kb}$ )

Additional file 6: Table S3. Significant variants in COL6A3 associated with the stages of lung cancer patients. (DOCX $25 \mathrm{~kb}$ )

Additional file 7: Table S4. COL6A3 haplotypes frequencies associated with lung cancer risk. (DOCX 18 kb)

Additional file 8: Table S5. In silico analysis for SNP function annotation. (DOCX $20 \mathrm{~kb})$

\section{Abbreviations}

95\%Cl: 95\% confidence interval; CMDs: Congenital muscular dystrophies; COL6A3: Collagen Type VI Alpha 3 Chain; HWE: Hardy-Weinberg Equilibrium; LD: Linkage disequilibrium; NSCLC: Non-small cell lung cancer; OR: Odds ratio; SCLC: Small cell lung cancer; SNPS: Single nucleotide polymorphisms 


\section{Authors' contributions}

YD and GW L completed genotyping and performed the manuscript. YS, JM W, ZC X and TB J participated in the statistical analysis of the data and modified the manuscript. MW C designed the study, co-supervised the work and modified the manuscript. All the authors have read and approved the final manuscript.

\section{Funding}

The study was supported by Scientific research and innovation fund of xianyang central hospital (2017 06).

\section{Ethics approval and consent to participate}

The study was approved by the Ethics Committee of Shaanxi Provincial Cancer Hospital and we obtained written informed consent from all individual participants.

\section{Consent for publication}

Not applicable.

\section{Competing interests}

The authors declare that they have no competing interests.

\section{Author details}

'Department of Respiratory Medicine, The First Affiliated Hospital of School of Medicine of Xi'an Jiao Tong University, \#277 Yanta West Road, Xi'an 710061, Shaanxi, China. ${ }^{2}$ Xianyang Central Hospital, Xianyang 712000, Shaanxi, China. ${ }^{3}$ Key Laboratory of Resource Biology and Biotechnology in Western China (Northwest University), Ministry of Education, Xi'an 710069, Shaanxi, China.

Received: 20 January 2019 Accepted: 26 June 2019

Published online: 08 July 2019

\section{References}

1. Cancer UK. Cancer stats: cancer statistics for the UK; 2012.

2. Chen W, Zheng R, Baade PD, Zhang S, Zeng H, Bray F, Jemal A, Yu XQ, He J. Cancer statistics in China, 2015. CA Cancer J Clin. 2016;66:115-32.

3. Spira A, Ettinger DS. Multidisciplinary management of lung cancer. N Engl J Med. 2004;350:379-92.

4. Ferlay J, Soerjomataram I, Dikshit R, Eser S, Mathers C, Rebelo M, Parkin DM, Forman D, Bray F. Cancer incidence and mortality worldwide: sources, methods and major patterns in GLOBOCAN 2012. Int J Cancer. 2015;136: E359-86.

5. Siegel RL, Miller KD, Jemal A. Cancer statistics, 2017. CA Cancer J Clin. 2017; 67:7-30.

6. Jemal A, Bray F, Center MM, Ferlay J, Ward E, Forman D. Global cancer statistics. CA Cancer J Clin. 2011;61:69-90.

7. Yokota J, Shiraishi K, Kohno T. Genetic basis for susceptibility to lung cancer: recent progress and future directions. Adv Cancer Res. 2010;109:51-72.

8. Rana M, Coshic P, Goswami R, Tyagi RK. Influence of a critical single nucleotide polymorphism on nuclear receptor PXR-promoter function. Cell Biol Int. 2017;41:570-6.

9. Hu QY, Jin TB, Wang L, Zhang L, Geng T, Liang G, Kang LL. Genetic variation in the TP63 gene is associated with lung cancer risk in the Han population. Tumour Biol. 2014;35:1863-6.

10. Jin X, Zhang KJ, Guo X, Myers R, Ye Z, Zhang ZP, Li XF, Yang HS, Xing JL. Fatty acid synthesis pathway genetic variants and clinical outcome of nonsmall cell lung cancer patients after surgery. Asian Pac J Cancer Prev. 2014; 15:7097-103.

11. Zazuli Z, Barliana MI, Mulyani UA, Perwitasari DA, Ng H, Abdulah R. Polymorphism of PXR gene associated with the increased risk of druginduced liver injury in Indonesian pulmonary tuberculosis patients. J Clin Pharm Ther. 2015;40:680-4.

12. Moon JY, Chang BC, Lee KE, Bang JS, Gwak HS. Effects of Pregnane X receptor genetic polymorphisms on stable warfarin doses. J Cardiovasc Pharmacol Ther. 2015;20:532-8.

13. Yamada Y, Kato K, Oguri M, Horibe H, Fujimaki T, Yasukochi Y, Takeuchi I, Sakuma J. Identification of 12 novel loci that confer susceptibility to earlyonset dyslipidemia. Int J Mol Med. 2019;43:57-82.

14. Gesta S, Guntur K, Majumdar ID, Akella S, Vishnudas VK, Sarangarajan R, Narain NR. Reduced expression of collagen VI alpha 3 (COL6A3) confers resistance to inflammation-induced MCP1 expression in adipocytes. Obesity (Silver Spring). 2016;24:1695-703

15. Yang J, Hou Z, Wang C, Wang H, Zhang H. Gene expression profiles reveal key genes for early diagnosis and treatment of adamantinomatous craniopharyngioma. Cancer Gene Ther. 2018;25:227-39.

16. Ao R, Guan L, Wang Y, Wang JN. Silencing of COL1A2, COL6A3, and THBS2 inhibits gastric cancer cell proliferation, migration, and invasion while promoting apoptosis through the PI3k-Akt signaling pathway. J Cell Biochem. 2018;119:4420-34.

17. Liu W, Li L, Ye H, Tao H, He H. Role of COL6A3 in colorectal cancer. Oncol Rep. 2018;39:2527-36.

\section{Publisher's Note}

Springer Nature remains neutral with regard to jurisdictional claims in published maps and institutional affiliations.
Ready to submit your research? Choose BMC and benefit from:

- fast, convenient online submission

- thorough peer review by experienced researchers in your field

- rapid publication on acceptance

- support for research data, including large and complex data types

- gold Open Access which fosters wider collaboration and increased citations

- maximum visibility for your research: over $100 \mathrm{M}$ website views per year

At $\mathrm{BMC}$, research is always in progress.

Learn more biomedcentral.com/submissions 